\title{
Un acteur incontournable de l'approvisionnement en fruits et légumes à La Réunion : les bazardiers de 1848 à aujourd'hui
}

A key player in fruit and vegetable supply in Reunion: the bazardiers from 1848

to today

Luca Piccin, Claire Cerdan, Claire Delfosse et Jean Paul Danflous

\section{(2) OpenEdition}

Journals

Édition électronique

URL : http://journals.openedition.org/geocarrefour/13580

DOI : $10.4000 /$ geocarrefour. 13580

ISSN : 1960-601X

Éditeur

Association des amis de la Revue de géographie de Lyon

Ce document vous est offert par CIRAD Centre de coopération internationale en recherche agronomique pour le développement

\section{cirad}

Référence électronique

Luca Piccin, Claire Cerdan, Claire Delfosse et Jean Paul Danflous, « Un acteur incontournable de l'approvisionnement en fruits et légumes à La Réunion : les bazardiers de 1848 à aujourd'hui », Géocarrefour [En ligne], 93/2 | 2019, mis en ligne le 17 janvier 2020, consulté le 13 février 2020. URL : http://journals.openedition.org/geocarrefour/13580; DOI : 10.4000/geocarrefour.13580

Ce document a été généré automatiquement le 13 février 2020.

(c) Géocarrefour 


\title{
Un acteur incontournable de l'approvisionnement en fruits et légumes à La Réunion : les bazardiers de 1848 à aujourd'hui
}

\author{
A key player in fruit and vegetable supply in Reunion: the bazardiers from 1848 \\ to today
}

Luca Piccin, Claire Cerdan, Claire Delfosse et Jean Paul Danflous

\section{Introduction}

1 Le développement économique extraverti qu'a connu La Réunion atteint aujourd'hui des limites qui se manifestent dans le secteur des fruits et légumes via une situation paradoxale que nous proposons de comprendre par une approche diachronique. Cet article se focalise sur les trajectoires de l'approvisionnement alimentaire à l'île de la Réunion en s'intéressant à une figure centrale du commerce insulaire : les bazardiers. Qui sont-ils? Depuis quand et comment investissent-ils les places de marché de l'île? Omniprésents, ils sont considérés par les institutions agricoles comme des éléments importuns : le dossier d'instruction des aides du POSEIDOM évoque un « marché informel encore important (bazardiers) $»^{1}$. Il en découle que l'analyse du marché des fruits et légumes est biaisée si on fait abstraction du rôle des bazardiers, et plus généralement du travail des intermédiaires, dans l'organisation des échanges.

2 Pour comprendre les processus qui amènent à la formation et à l'évolution d'un marché des fruits et légumes marquées par la prégnance des bazardiers, notre analyse se fonde sur les concepts de la géographie des transitions et de la perspective multi-niveaux (Hansen et Coenen, 2015; Boschma et al, 2017). Cette dernière souligne l'existence de configurations d'acteurs, de technologies et de règles (routines cognitives, croyances, modes de vie et habitudes de consommation, réglementations, etc.) qui orientent les 
activités humaines et se reproduisent à travers elles. La notion de dépendance du sentier y occupe une place centrale : elle postule que les choix actuels sont fortement contraints par les choix du passé. Des nouvelles trajectoires apparaissent quand les innovations qui émergent au niveau des niches et les changements du paysage sociotechnique (éléments de contexte plus larges sur lesquels les acteurs n'ont pas d'influence sur le court terme, comme guerres, évolution culturelle ou changement climatique) créent des pressions "verticales », ouvrant des fenêtres d'opportunité pour des niches d'innovation qui peuvent ainsi défier la stabilité du régime sociotechnique (Geels et Schot, 2007). Nous défendons une approche pragmatique des transitions, où les acteurs "font avec l'espace", c'est-à-dire où l'espace n'est plus conceptualisé comme une structure absolue ou relative, mais se construit pendant l'action (Lussault et Stock, 2010). Nous cherchons par là à dépasser certaines limites qui caractérisent l'approche des transitions sociotechniques, pour restituer toute la complexité systémique des changements, les recompositions des rapports de pouvoir et les controverses, en plaçant le regard là où les marchés se matérialisent; ce qui interroge la question de "l'appropriation» professionnelle et sociale d'espaces marchands fragiles et chargés de qualités et valeurs complexes (Lamine et al, 2015 ; Leblanc, 2017).

3 Les résultats présentés dans cet article proviennent d'un corpus de données comprenant : une bibliographie approfondie sur l'histoire locale ; archives de la presse régionale (plus de 300 articles entre 1983 et 2003 dans : Le Quotidien, Le Journal de l'Ile de La Réunion, et en moindre mesure Témoignages) ; littérature grise (rapports d'études et documents techniques de collectivités locales, instituts de recherche et développement et chambres consulaires). Une série d'entretiens $(n=60)$ réalisés dans le cadre d'une thèse de doctorat entre décembre 2016 et août 2018 auprès des acteurs du marché des fruits et légumes ont permis de clarifier l'évolution récente.

4 L'article est structuré en trois parties. Dans la première nous montrons comment pendant longtemps de nombreuses espèces fruitières et potagères sont cultivées dans les «jardins créoles» pour la subsistance mais aussi pour la commercialisation. Des espaces marchands se construisent peu à peu autour de commerçants: marchands ambulants appelés bazardiers et petites boutiques tenues par la communauté chinoise. La deuxième partie se focalise sur la transition correspondante à la départementalisation, pendant laquelle les autorités locales tentent de réduire les innombrables intermédiaires accusés de parasiter le marché par l'organisation collective des ventes via les coopératives. Il en résulte un système d'intermédiation complexe dont les évolutions restent difficiles à saisir (troisième partie).

\section{Emergence et affirmation des premières formes marchandes dans la période coloniale}

5 L'abolition de l'esclavage en 1848 accélère le passage à un mode de production capitaliste basé sur le sucre, fondé sur : la substitution des travailleurs engagées aux esclaves, la marginalisation sur des espaces moins productifs des classes subalternes et une pression très forte sur les ressources naturelles. Ces facteurs participent à ouvrir une crise entre 1860 et 1881, aggravée par la concurrence des autres colonies et des betteraviers. Au niveau du paysage sociotechnique, un véritable choc est représenté par la dénonciation, par l'empire britannique en 1882, de la convention qui réglementait le recrutement à La Réunion de travailleurs indiens engagés : c'est la fin 
du «salariat contraint » et donc de la principale source de main d'œuvre. Le régime politique réagit en attachant à la terre les colons via le système du " colonat partiaire ", mode de production apparu à La Réunion dans la seconde moitié du XVIII ${ }^{e}$ siècle : le propriétaire loue une parcelle à un colon, lui fournit les intrants et lui impose les choix de culture, avec un partage des récoltes $1 / 3-2 / 3$ ou $1 / 4-3 / 4$. La culture du sucre est alors maintenue, mais le manque de main d'œuvre prolonge la crise jusqu'en 1914 (Ho, 2004). La transition au capitalisme se met en place dans ce cadre, sans bouleverser l'organisation socio-économique du territoire.

6 Les pratiques alimentaires des populations reflètent cette organisation (Eve, 2013). Trois modèles alimentaires se côtoient : le premier est celui des esclaves, dominé par le maïs et les racines (manioc, patates), relayés par le riz, réclamé par les engagés asiatiques. Les légumineuses fournissent les protéines, parfois accompagnées de morue séchée. Le piment et les brèdes ${ }^{2}$ viennent compléter l'assiette. Le second est celui des gens pauvres, esclaves affranchis ou Petits Blancs des Hauts ${ }^{3}$, similaire au précédent, mais où des mélanges apparaissent plus souvent, avec viandes et poissons, reposant sur la débrouillardise et la récupération. Le troisième modèle est celui des propriétaires libres et aisés, caractérisé par la diversité. Il diffère des autres par une recherche du goût très poussée : c'est ici que s'élaborent potages, fricassés, daubes, fritures, rôtis, caris, civets, et pâtisseries.

\section{La nécessité de nourrir une population croissante pousse l'autorité à mettre en place des dispositifs marchands}

7 Dans l'organisation sociale coloniale la production des denrées est faite par le personnel qui travaille dans les domaines des propriétaires fonciers, au détriment des échanges marchands. Les catégories subalternes sont laissées pour compte et se débrouillent en cultivant un petit lopin ou s'adonnant aux vols. Une forme d'habitat créole, fondée sur le triptyque : case, kour et jardin, se diffuse sur l'île; traversant les catégories ethnoculturelles et les frontières entre urbain et rural, elle va marquer le mode de vie réunionnais jusqu'aux années 1980 . 
Figure 1 : Pendant plusieurs siècles la production vivrière est partie intégrante de l'habitat créole

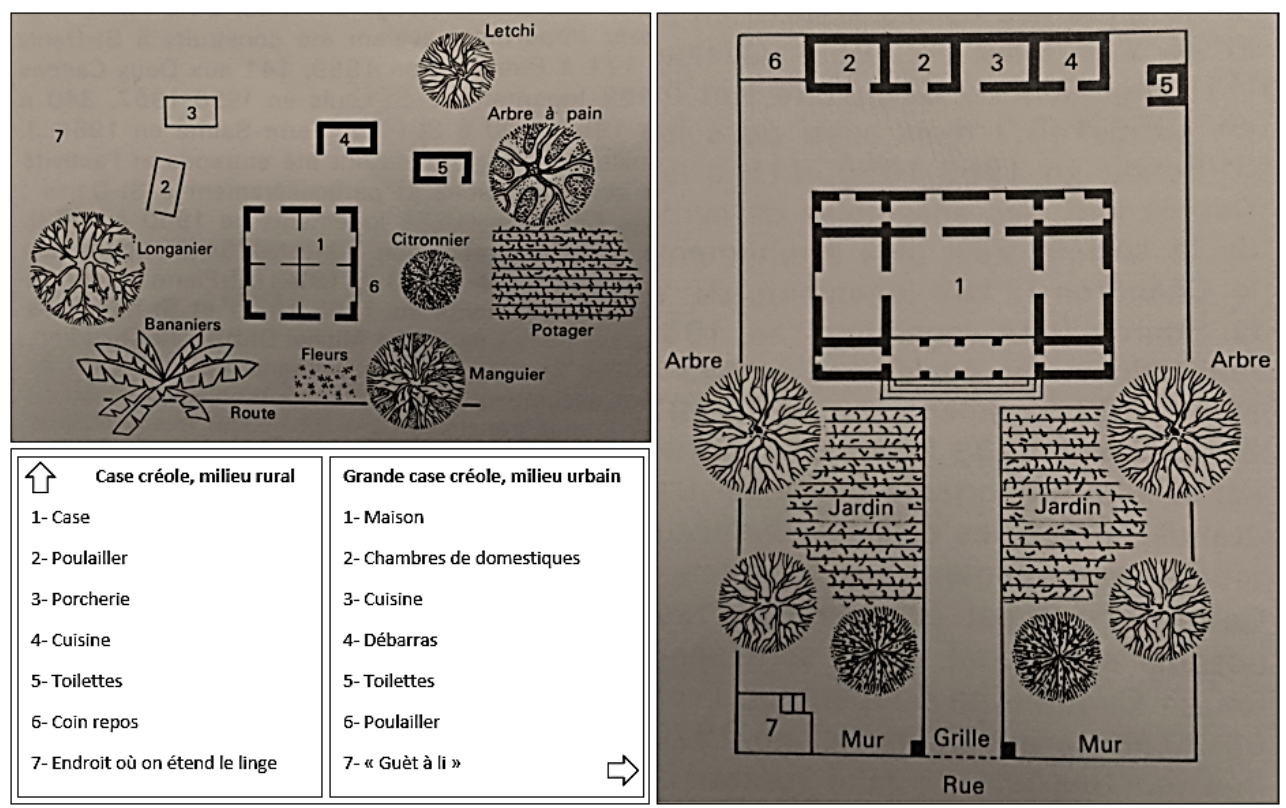

Lecompte, 1975

8 Malgré la primauté de l'économie de subsistance, les salaires versés aux travailleurs engagés, bien que très bas, permettent l'apparition d'une demande solvable, ce qui pousse certains paysans à céder des excédents aux bazardiers, mal perçus par la plantocratie. Dans un contexte de pénurie alimentaire permanente, les dizaines de milliers d'individus introduits de force sur l'île posent des problèmes d'ordre public. Antoine Fitau, conseiller colonial de 1834 à 1848, réclame « d'efficaces mesures de police à l'égard des bazardiers » (Fitau, 1859, p. 5-6), ce qui atteste d'une présence déjà importante de ces derniers à la moitié du siècle. En grande majorité pauvres vendeurs à la sauvette qui attendent une pièce de monnaie en échange d'un rafraîchissement, certains sont néanmoins capables de s'organiser pour répondre à la demande émergeante, comme au Grand Marché de Saint-Denis. Bâti sur le modèle des halles parisiennes, il constitue le premier dispositif mis en place par les autorités afin de réguler les transactions marchandes. En 1861 cet espace était déjà fréquenté par des marchands d'origine indienne; Louis Simonin s'y réfère lors de son séjour à Saint Denis (Simonin, 2007, p. 13). Jean Defos du Rau (1960, p. 373) en parle dans un passage où il explique comment le village de la Possession est :

« né des relations entre Saint-Paul et Saint-Denis ; un service de barques assurait la liaison par mer au long de la falaise ; relais et auberges étaient fréquentés aussi par les cavaliers franchissant péniblement la Montagne ; il fut un temps (1830-1880) où 15 péniches y embarquaient de nuit 80 à 100 bazardiers [...], allant ravitailler la capitale ». 
Figure 2 : L'approvisionnement des principales villes de La Réunion à la fin du XIXe siècle

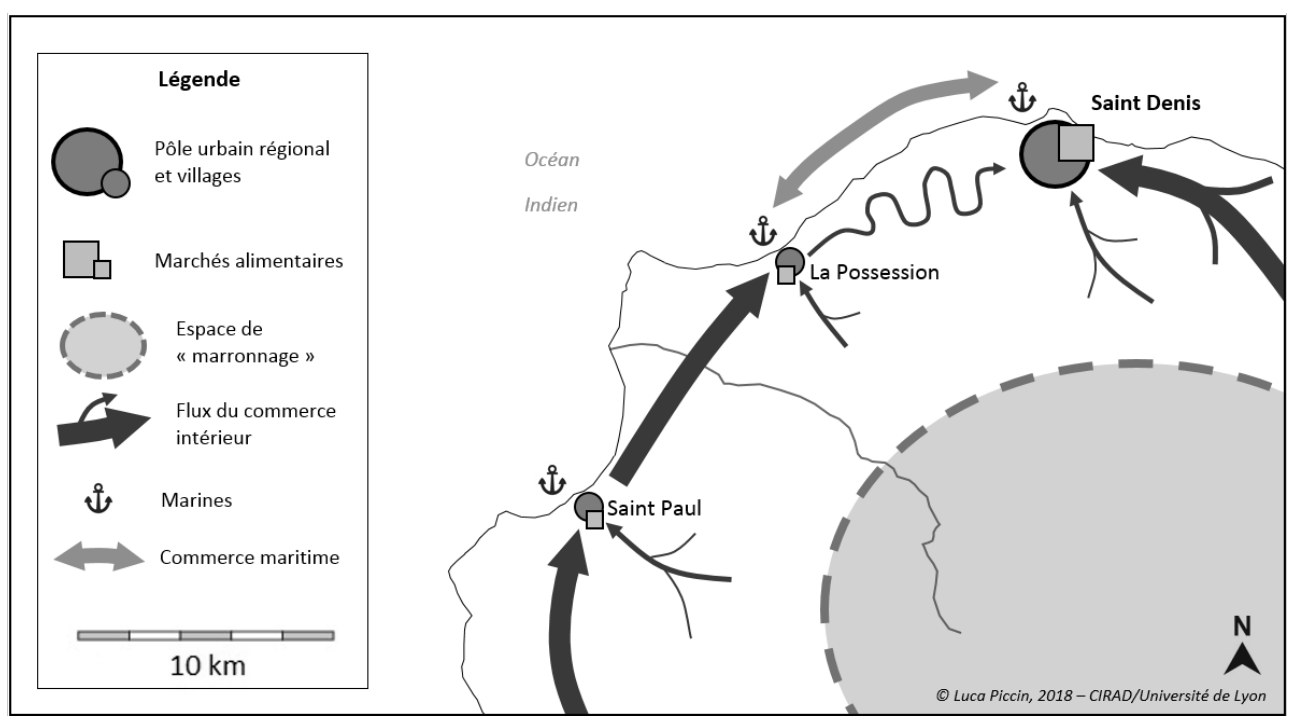

Malgré les risques, ce commerce maritime est lucratif et des Indiens s'associant entre eux parviennent par ce biais à avoir le monopole des fruits et légumes (Ramsamy, 2012). Certains d'entre eux sont même capables de concurrencer les entreprises de batelage introduites quelques décennies plus tôt par les grands propriétaires ${ }^{4}$. On peut affirmer que des bazardiers indiens ont participé à l'organisation du marché des fruits et légumes via la mise en place d'un service de batelage original, liaison nécessaire pour surmonter l'obstacle géographique de la falaise qui sépare Saint-Paul et Saint-Denis. Les Indiens étaient capables de faire preuve d'esprit entrepreneurial, et n'étaient pas seulement des intermédiaires parasites, comme le voudrait une idée reçue répandue dans les colonies insulaires (Mintz, 1964).

Le batelage ne disparait définitivement qu'après la Première guerre mondiale, au profit du «ti train » qui, entre 1882 et 1976, assure la liaison entre Saint-Benoit à l'Est et Saint-Pierre dans le Sud, en passant par Saint-Denis. La réalisation des ports de la Pointe des Galets et de Saint-Pierre, conjointement à celle du chemin de fer, mettront fin à ce commerce côtier à la faveur des échanges sur terre. Ces chantiers publics permettent aussi d'injecter de la monnaie dans une économie qui en avait fort besoin (Ho, 2008). Il est incontestable que le chemin de fer a joué un rôle primordial dans la percée des marchands indiens et chinois dans l'Est et dans le Sud, tout en favorisant la circulation et l'écoulement de produits agricoles de la part des bazardiers, notamment en proximité des gares.

\section{Le développement des boutiques chinoises, pivot du commerce alimentaire réunionnais}

11 Le choix de la spécialisation sucrière implique de développer un commerce d'importexport. A la fin des années 1850 la première boutique chinoise ouvre à la Possession. L'arrivée des engagés chinois va propulser ce petit commerce d'abord dans les centresvilles puis dans les écarts: les Chinois refusent systématiquement de travailler aux conditions imposées par les sucriers. C'est à partir de 1862, avec l'autorisation de l'immigration libre, que leur nombre ne cesse d'augmenter et les boutiques occupent tous les carrefours des routes. De 11 petits commerces chinois en 1861, ils passent à 70 
au début du XXe siècle. En 1911, sur 1160 Chinois installés dans l'île, 957 personnes, soit 85 \% se déclarent commerçants (Wong Hee Kam, 1996). Dans la ville de Saint-Denis, le nombre de détaillants chinois est déjà supérieur à celui de leurs homologues français en 1915. En dépit des attaques dans la presse et du racisme ambiant, les chinois finissent par conquérir un créneau qui permettra leur insertion socio-économique. Cette réussite tient à des multiples facteurs :

12 - les Chinois qui arrivent à La Réunion, notamment les immigrants libres, ne sont pas des paysans. Dans la plupart des cas ils ont déjà les rudiments du commerce et savent se servir d'innovations comme les insertions publicitaires ou le boulier. Certains d'entre eux viennent sur l'Ile pour rejoindre leur famille, après être passés par l'île Maurice, Madagascar, ou Batavia 5 .

13 - des solidarités familiales et ethniques se mettent en place, et des structures associatives voient le jour. Parmi celles-ci il faut mentionner la Chambre de Commerce Chinoise, fondée en 1916 par Akwon Lawson, malgré les hostilités de la plantocratie. Elle va fonctionner jusqu'à la fin de la Seconde guerre mondiale agissant comme consulat, agent commercial et soutien à la communauté chinoise, épaulée par le Guomindang: le parti communiste chinois. Des liens communautaires très forts ont permis aux immigrants chinois de mutualiser des moyens, de s'entraider lors de périodes difficiles et de maintenir leurs spécificités culturelles, au point qu'on comptait douze école primaires chinoises en 1943 sur l'île.

14 - au niveau culturel, outre le lien à la terre qui était très fort chez les Indiens, une certaine proximité avec l'Inde avait sans doute joué un rôle au niveau alimentaire. Comme l'a montré Pierre Gourou (1984), malgré l'appartenance à des aires de civilisation différentes, l'utilisation du riz comme aliment de base est une constante que l'on retrouve de la Chine méridionale à Madagascar, en passant par la péninsule indochinoise et l'Inde. Les engagés indiens ont toujours revendiqué et obtenu une nourriture différente par rapport à celle de leurs prédécesseurs africains (riz au lieu du manioc ou du maïs). En période de crise il est donc probable que les engagés étrangers ait préféré se tourner vers les boutiquiers où ils trouvaient des aliments convenables (riz, mais aussi des ingrédients moins usités chez les marchands d'origine française, comme racines ou épices tels le gingembre, le curcuma, etc.).

15 - enfin, dans le bagage socio-culturel que les Chinois ont amené avec eux figure aussi une éthique du travail ${ }^{6}$. Les Chinois sont des travailleurs acharnés et minutieux.

16 Un dernier facteur réside dans le système de crédit appelé " roulement ». Jusqu'à la fin des années 1950, le bon de sucre ${ }^{7}$ remis aux planteurs par les usiniers constitue une monnaie scripturale parallèle au franc CFA, ce qui fait des commerçants chinois des proto-banquiers. Alors que la plupart des ventes s'effectuent uniquement à crédit, autant chez les classes défavorisées que dans les couches sociales les plus aisées, le chinois se trouve dans une position stratégiquement favorable, du fait de ses rapports privilégiés avec le négoce et les importateurs. Les boutiques font de l'avance et permettent à de nombreux ménages d'attendre la fin du mois avec moins de peine. L'argent-carnet vient donc au secours de la population en attendant le revenu des récoltes. Pour le marchand, cette pratique est aussi et surtout un moyen efficace de conserver sa clientèle. 


\section{Le commerce alimentaire comme voie d'insertion socio-économique dans une île hostile}

En favorisant l'extroversion de l'économie insulaire, le régime colonial est responsable d'un état de crise chronique et de pénuries alimentaires fréquentes. Comme l'a démontré Ho Hai Quang (2008), jusqu'aux années 1960 le régime d'accumulation se caractérise par un découplage entre le système capitaliste reposant sur l'agro-export et l'économie domestique. Qu'ils soient colons ou propriétaires d'une parcelle, les paysans sont surexploités, puisque les salaires versés ne sont qu'un complément aux activités agricoles qu'ils mènent parallèlement à celles qui sont exigées par la plantocratie. Paradoxalement, cela ouvre des fenêtres d'opportunité pour les niches du commerce alimentaire. On assiste à une reconfiguration progressive de l'approvisionnement, qui passe d'un système entièrement autarcique à une agriculture de subsistance avec écoulement marchand du surplus, complémentaire aux productions industrielles vouées à l'export.

Figure 3 : Représentation graphique de la reconfiguration de l'approvisionnement

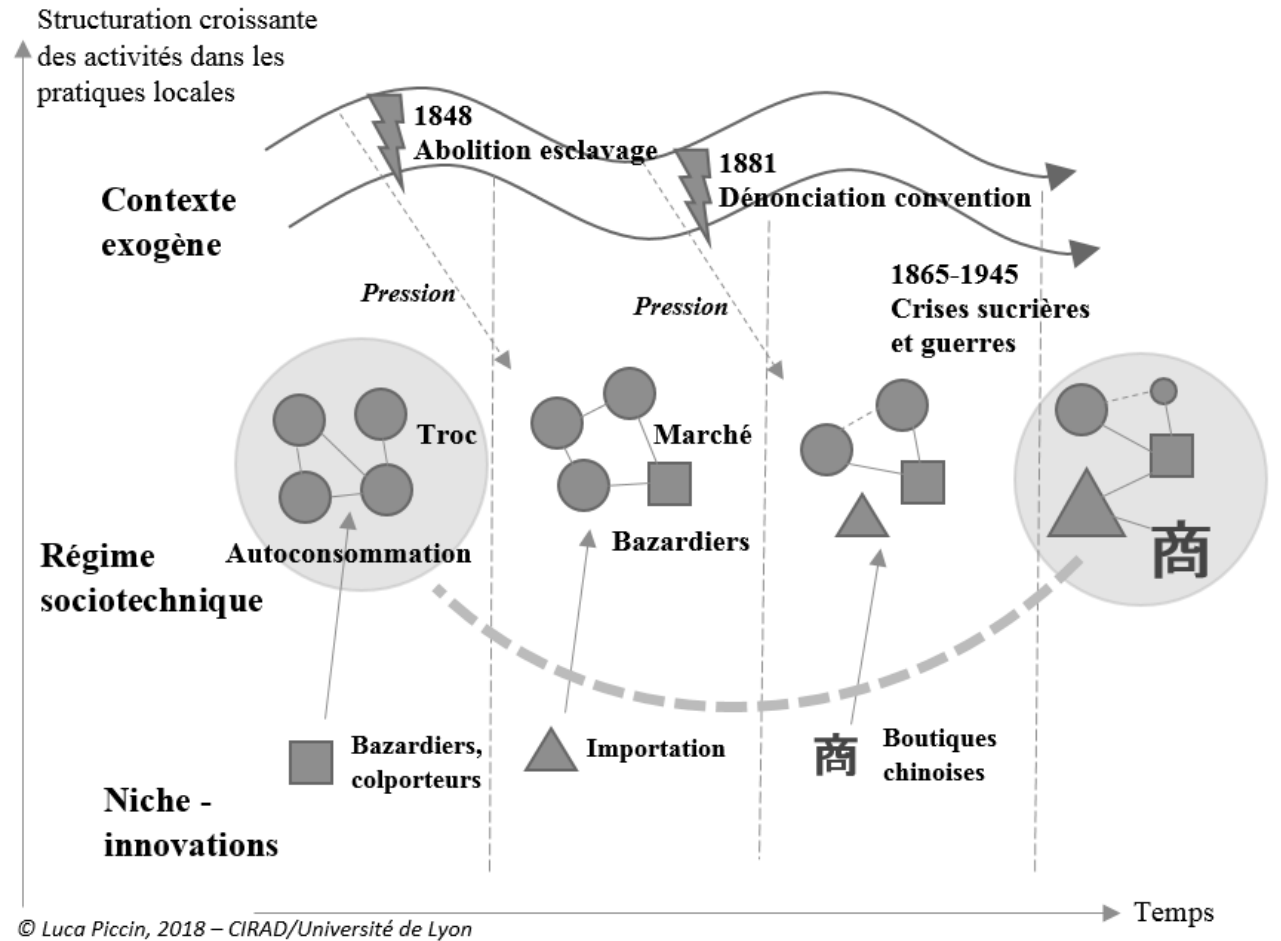

alimentaire

Jusqu'à la fin des années 1950 l'essentiel de la production est consommé au sein du ménage ou du quartier, à travers des relations de réciprocité. L'écoulement des surplus éventuels se fait toujours par l'intermédiaire des bazardiers indiens et/ou des commerçants chinois (Dupon, 1967). Les produits agricoles se vendent sur les marchés couverts des principales villes côtières, au bord de rue à la sauvette, dans les boutiques, ou encore de manière intermittente dans les zones de villégiature. A partir des années 1960 la départementalisation transforme l'approvisionnement alimentaire, mais la position des bazardiers dans le commerce des fruits et légumes et celle des Chinois dans le commerce de détail est solidement acquise. 
Les marchands affluent alors dans les villes à bord du train ou tout simplement à pied. Le témoignage d'Acadius Mézino, ancien habitant de la commune de Petite-Ile, évoque des bazardiers indiens qui, entre les années 1930 et les années 1950, livrent du pain depuis Saint-Pierre en échange de bouteilles vides ou de flacons qu'ils remettent à d'autres commerçants ou aux pharmacies. Équipés d'un «tacon », barre en bois portée sur les épaules avec deux sacs accrochés aux extrémités, ces colporteurs passaient de cour en cour pour acheter œufs et volailles et les revendre le lendemain à Saint-Pierre (Bouchet, 2009, p. 246). Les Indiens ont su profiter de la niche du commerce alimentaire, mais la pratique du bazar est courante aussi chez les Petits Blancs des Hauts. Dans le cirque de Grand Bassin par exemple :

« De chez nous jusqu'au Haut $23^{8}$, il fallait suivre une ligne-sentier, dans le rempart là où la montagne se casse tout droit jusque vers le bas. Il y avait à peu près quatre kilomètres de montée, et mon père y allait chaque semaine, tout seul. Il partait pour la journée avec un panier sur la tête. Il portait quelques légumes à la boutique, parfois un ou deux poulets, et même des fruits, à la saison. Et il redescendait de la boutique: les allumettes, le sel, le savon, parfois un peu de bonbons pour nous. Toute la semaine, il faisait de l'élevage, avec nous et avec ma mère, et il cultivait dans le Fond, bien plus bas, une petite portion de terre qu'il avait reçue de son père. Et toutes les semaines c'était comme ça. Il y avait des fruits, des racines, du maïs. On vendait les haricots. Et mon père tuait son cochon, et alors il en montait les bons morceaux à la boutique. On n'était pas malheureux : on mangeait à sa faim, on était tranquilles » (Benoist, 1981, p. 26-27).

Comme dans l'Afrique coloniale, à La Réunion l'intermédiation marchande entre les espaces agricoles pourvoyeurs de vivres et les espaces urbains demandeurs est tributaire des activités de transport (Chaléard et al, 2002), extrêmement difficiles à La Réunion. Dans le cas du batelage, les conditions climatiques rendaient la navigation dangereuse et les chaloupes vulnérables; pour les villages de l'intérieur de l'île, c'est le relief très accidenté qui rendait difficile l'approvisionnement. Dans ce contexte défavorable, exacerbé par les critiques racistes que la presse du régime politique dirigeait envers les étrangers, les catégories subalternes des bazardiers, mais aussi des boutiquiers chinois, réussissent une insertion socio-économique remarquable. En utilisant des moyens de fortune, ou tout simplement par la force de leurs membres, ils parviennent à établir des flux de marchandises certes réduits en volumes, mais pourtant essentiels au fonctionnement de l'économie alimentaire. 
Figure 4 : Bazardiers entre 1900 et 1920, sous l'œil vigilant d'un officier de police

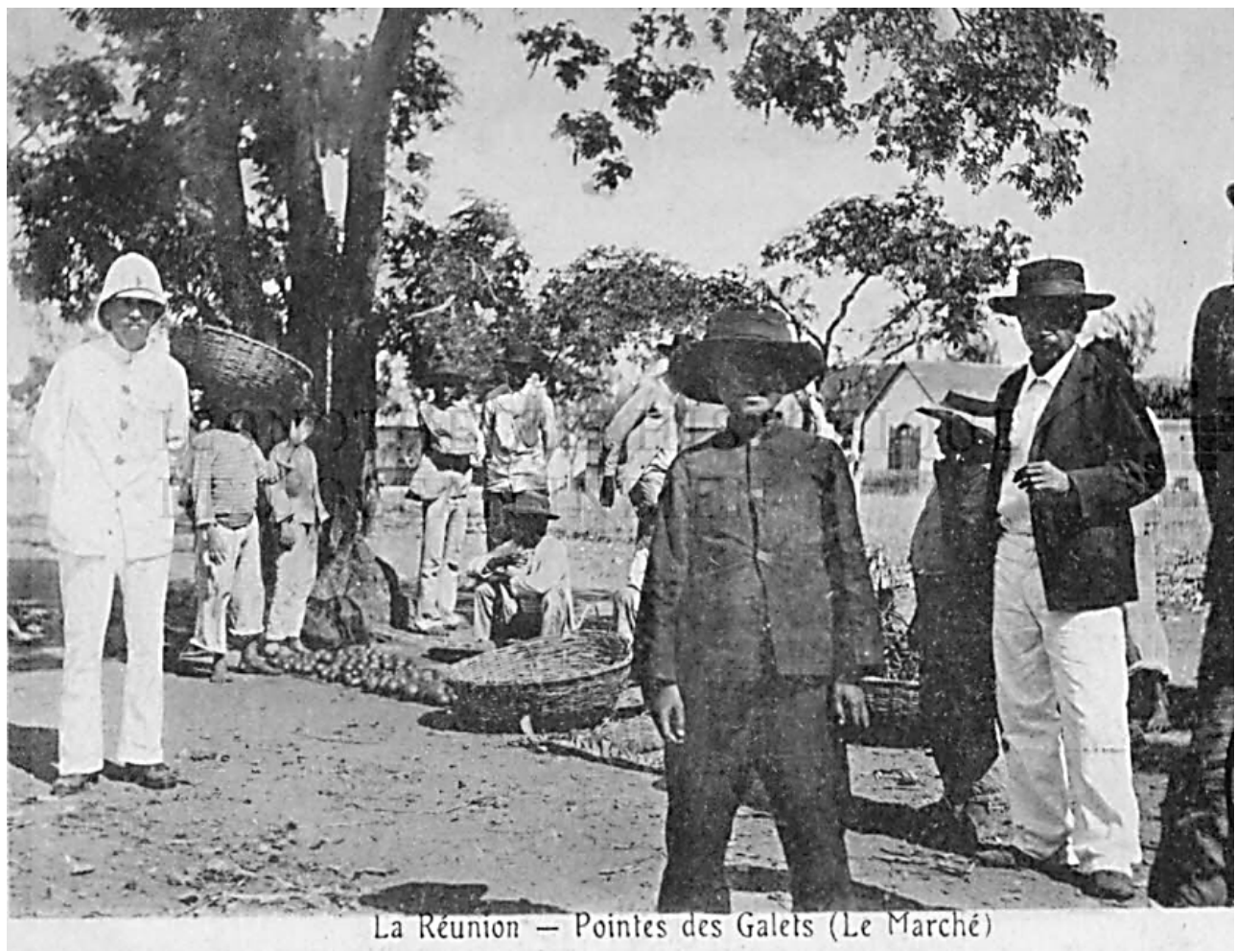

Carte postale, collection Jean-François Hibon de Frohen

\section{L'approvisionnement en fruits et légumes à l'heure de la grande transformation du système alimentaire}

La Réunion devient département français en 1946, puis région en 1982 et est progressivement intégrée à l'espace économique européen jusqu'à devenir une région ultrapériphérique européenne dans les années 1990. Ce double statut apporte à l'Ile d'importants crédits publics qui impactent profondément la trajectoire de développement. L'économie de plantation est remplacée par une économie de services marchands et non marchands et l'émergence d'une nouvelle classe sociale s'interposant entre les héritiers de la plantocratie et le prolétariat.

Figure 5 : Indicateurs de l'intervention étatique dans l'évolution de l'économie insulaire

\begin{tabular}{|c|c|c|c|c|c|c|c|c|c|}
\hline & 1954 & 1961 & 1967 & & 1974 & & 1982 & \multicolumn{2}{|c|}{1990} \\
\hline Primaire \% & 54 & 44 & 30 & & 21 & & 15 & \multicolumn{2}{|c|}{8} \\
\hline Secondaire \% & 25 & 21 & 22 & & 19 & & 17 & \multicolumn{2}{|c|}{19} \\
\hline Tertiaire \% & 21 & 35 & 48 & & 60 & & 68 & \multicolumn{2}{|c|}{73} \\
\hline \multicolumn{4}{|c|}{ Part des prestations sociales / revenu des ménages } & \multicolumn{6}{|c|}{ Transferts publics depuis la métropole* } \\
\hline & & 1961 & 1995 & \multicolumn{2}{|c|}{1970} & & 80 & \multicolumn{2}{|c|}{1990} \\
\hline Réunion & & $15 \%$ & $45 \%$ & \multicolumn{2}{|c|}{828,6} & & 57,7 & \multicolumn{2}{|c|}{12538} \\
\hline Métropole & & $20 \%$ & $36 \%$ & $\% \mathrm{PIB}$ & 59,1 & $\% \mathrm{PIB}$ & 61,7 & $\% \mathrm{PIB}$ & 60,8 \\
\hline
\end{tabular}

* En millions de Francs 
Alors qu'on assiste à un processus d'assimilation culturelle et à une progressive amélioration du revenu moyen, la modernisation capitaliste de l'économie ainsi que l'héritage de la domination coloniale s'accompagnent d'un chômage chronique et d'un haut niveau d'exclusion. Pour attirer des fonctionnaires métropolitains, l'administration leur propose des majorations de salaire. Il faut attendre 1995 pour que le salaire minimum atteigne la même valeur qu'en métropole ${ }^{9}$. L'urbanisation favorisée par une politique de défiscalisation et la pression démographique posent enfin le problème de l'aménagement d'un territoire insulaire étroit, saturé et déséquilibré (Coder et al, 2016).

Soumise aux lois d'orientation agricole, l'agriculture réunionnaise a connu une évolution originale depuis les années 1960 jusqu'aux années 1990 avec la diffusion massive d'un modèle d'exploitation familiale en faire-valoir direct, se substituant à de grandes plantations exploitées principalement en colonage. Résultat de la réforme foncière menée par la SAFER et le Crédit Agricole, cette réattribution correspond aussi au désengagement progressif du capital du secteur agricole vers le secteur des services (Ho, 2011). Cette politique des structures est complétée par une politique volontariste de soutiens directs aux productions et de régulation des marchés pour la canne à sucre et les productions animales. Pour les fruits et légumes, les choses sont moins simples : la conjonction du caractère périurbain de l'agriculture réunionnaise avec les contraintes d'insularité, de saisonnalité et de variabilité, révèle d'un côté les échecs multiples des tentatives de standardisation de l'offre et de la demande et de l'autre, l'adaptation des bazardiers et des agriculteurs aux mutations territoriales.

\section{Agriculteurs et commerçants face au projet modernisateur de l'économie}

Pour concrétiser le projet modernisateur, les autorités font appel à des ingénieurs agronomes, regroupés dans un Institut de Recherches sur les Fruitiers et les Agrumes (IRFA) et chargés d'accompagner le processus de diversification de l'économie agricole. Constatant que "les marchés sont inorganisés ", les modernisateurs s'attachent à créer des structures coopératives, véritables plaques tournantes permettant la gestion centralisée des ventes et la standardisation des pratiques et des produits. Notons que cette stratégie a été appliquée sans succès dans d'autres anciennes colonies, comme en Algérie, où Bernard de Raymond (2013, p. 108) a démontré comment la nécessité de faire passer les produits par un canal unique, une filière, «à la manière des oléoducs ou des gazoducs ", ne va pas de soi, car la logique de la "fluidité industrielle ", s'adapte mal à la variabilité et à la saisonnalité des fruits et légumes. Cela est d'autant plus évident à La Réunion, où la diversité bio-culturelle ${ }^{10}$ est extrême et où la consommation de fruits et légumes est traditionnellement liée à l'autoproduction et donc à l'habitat. De ce fait, le projet des modernisateurs implique aussi une standardisation des modes de consommation et des modes de vie. La politique d'éradication des bidonvilles au profit des grands ensembles sera aussi une politique de transformation du mode de vie, d'effacement du jardin et du verger créole, dédaignant un habitat pourtant reconnu comme « le pivot central de l'art de vivre créole » (Jauze, 2000, p. 18).

Les agronomes de l'IRFA considèrent que pour un meilleur équilibre entre milieu rural et milieu urbain il faut agir sur le prix de revient, qui doit s'aligner sur les prix des produits importés via des productions intensives et spécialisées, autrement dit «en 
faisant ressortir les vergers dignes de ce nom et les vergers créoles et isolés » (IRFA, 1979, p. 12). Les cours élevés constatés sur les marchés sont expliqués uniquement par les spéculations des intermédiaires, certes réelles, mais liées aussi à la structure très inégalitaire des revenus. Nous savons que la demande captive, en particulier en milieu urbain, disposait de revenus élevés du fait des primes à l'éloignement et des majorations de salaire. Dans ce contexte, les frontières entre agriculteurs et bazardiers sont très difficiles à discerner : au début des années 1980, les techniciens de la Chambre d'Agriculture estiment que pour faire face à la faiblesse des revenus, au moins un agriculteur sur quatre est aussi un bazardier ${ }^{11}$.

Une enquête menée par la Direction Départementale de l'Agriculture en 1984 reconnait la pluralité des formes de mise en marché et établit cinq axes pour l'avenir de la commercialisation : premièrement la création de deux marchés de gros (un dans le Sud et un dans l'Est). Le soutien public est maintenu pour: a) les organisations de producteurs et $b$ ) l'exportation. Pour les petits paysans sans véhicule est envisagée la création de petits groupements, afin de mutualiser le moyen de transport et améliorer gamme et quantité des produits. Enfin, l'autorité préconise d'améliorer et de multiplier un dispositif émergeant: le marché forain ${ }^{12}$. Pour éviter les spéculations opérées par certains bazardiers qui ont une fonction de grossistes, des agriculteurs réclament et obtiennent à partir des années 1970 la création des premiers marchés forains qui leur permettront de vendre directement au détail. Ces dispositifs marchands ont un franc succès et se multiplient sur tout le territoire : les premiers sont créés à Saint-Denis et à Saint-Paul. 15 marchés forains existent sur l'île en 1980 ; ils sont une trentaine aujourd'hui. Contrairement à la métropole, les marchés forains sont à La Réunion des dispositifs innovants, qui émergent pour permettre la rencontre d'une offre et d'une demande spécifiques.

Figure 6 : Gauche : le nouveau marché forain du Chaudron, à Saint-Denis. Droite : l'essor de l'import-distribution et la création d'infrastructures routières favorisent la motorisation d'agriculteurs et bazardiers

Source : Le Quotidien, 29 janvier 1985

\section{La parabole de la SICAMA, ou l'échec d'une politique de standardisation de la production et de la vente de fruits et légumes}

Les responsables agricoles réunissent des paysans illettrés ou analphabètes le 23 avril 1972, face au court-métrage : «Une Sica pour quoi faire?», dans lequel est montré l'exemple d'un producteur maraîcher qui cherche à vendre sa production et rencontre les responsables de la SICA Maraichère, qui lui expliquent que le système coopératif intéresse des producteurs réguliers. Le mois suivant, l'expérience est répétée avec le film: «Seul ou avec les autres?». Ces projections attirent respectivement 95 et 88 groupes, soit 1298 et 1026 participants (Nicaise, 2013). Parmi ces participants, beaucoup finiront par rejoindre la SICAMA, qui regroupera 1000 adhérents quelques années après. Malgré ces efforts, le dispositif peine à séduire. La presse du 8 janvier 1985 donne la parole à un paysan de l'Ouest qui loue un emplacement au marché de Saint Denis :

« Si on réussit à planter, on réussit à vendre quel que soit le prix [...]. Les bazardiers passent tous les jours pour acheter des produits dans la région mais je ne veux plus passer par eux. Quand je leur vends un citron à un franc, ils le revendent à trois pour dix francs sans avoir rien eu à faire alors que je passe toute l'année dans mon 
verger. Je préfère aller les vendre moi-même à deux francs et ils s'écoulent très bien. Certains commerçants, en ne pensant qu'à leur profit immédiat bloquent la consommation de produits locaux, et donc le développement de nos exploitations [...] Les coopératives sont des organismes hypertrophiés où il y a trop de personnel et où il faut des produits calibrés. On est plus motivés quand on fait tout par soi-même... Mais il faut quand même de tels organismes, car tout le monde ne peut être producteur et vendre en même temps. Cela permet aux agriculteurs d'écouler leurs productions ».

La SICAMA fournissait des collectivités (écoles, hôpitaux) dès sa création en 1963, et depuis la fin des années 1970 avait ouvert un créneau pour l'exportation de fruits. Auparavant considérée comme «la poubelle du département ", elle fait transiter dans ses structures environ 6000 tonnes de produits, soit $26 \%$ de la production insulaire en $1983^{13}$. Pour les autorités ce n'est pas suffisant : les «Etats Généraux du développement agricole », le 14 décembre 1982, regrettent que la diversification soit entravée par "l'anarchie du marché des fruits et légumes $»^{14}$. Un pas décisif vers l'approche par filière s'opère avec le vote du budget du Conseil Général en 1984. Il est décidé de financer directement les structures coopératives, sans passer par la Chambre d'Agriculture qui s'occupait jusque-là de redistribuer les aides publiques. Sont mis en avant les agriculteurs qui acceptent la discipline de l'organisation collective: pour chaque filière retenue, le groupement passe une convention avec le Conseil Général qui précise les actions à mener ainsi qu'une liste d'agents chargés de les mettre en œuvre. Cette option est contestée, notamment par le groupe communiste :

«Avec l'approche par filière, on dépouille la Chambre d'Agriculture (...) On risque également d'ignorer la masse et de privilégier ceux qui s'en sortent déjà. Ce sont des orientations très précises que nous ne pouvons pas passer sous silence ${ }^{15}$.

Alors que cette approche est adoptée, l'évolution du marché va dans une direction opposée : en mai 1987, la SICAMA est en redressement judiciaire. Endettée, la société qui devait organiser les ventes de fruits et légumes est incapable d'honorer ses créances. On découvre alors que sur un fichier de 1100 adhérents, seulement 100 à 200 livrent régulièrement leur production ${ }^{16}$. Le tribunal du commerce avec une décision du 10 juin 1987 va confier la gérance de la structure à un grossiste : la Société Primeur Réunion. Un bazardier se trouve ainsi à la tête d'un dispositif collectif. Une des raisons des échecs de la SICAMA (et des structures qui la remplaceront dans les années 1990) réside dans le non-respect de l'obligation d'apport total de la part de nombreux adhérents. Un problème encore actuel, pourtant connu depuis les années 1970 :

«Le planteur ou éleveur a tendance à vouloir écouler par l'intermédiaire de ces organismes que des produits de moins bonne qualité qu'il ne peut vendre ailleurs à un prix aussi intéressant [...] ou à vouloir obtenir un prix plancher pour écouler sa production en période de surproduction sans consentir un prix plafond quand il en manque sur le marché. Quant aux directions des SICA elles n'estiment pas possible la signature de contrats de livraison prix garantis pour leurs clients » (Lefèvre, 1974, p. 340). 
Figure 7 : La transformation de l'approvisionnement avec l'affirmation de nouveaux circuits marchands au détriment de l'autoconsommation et des boutiques.

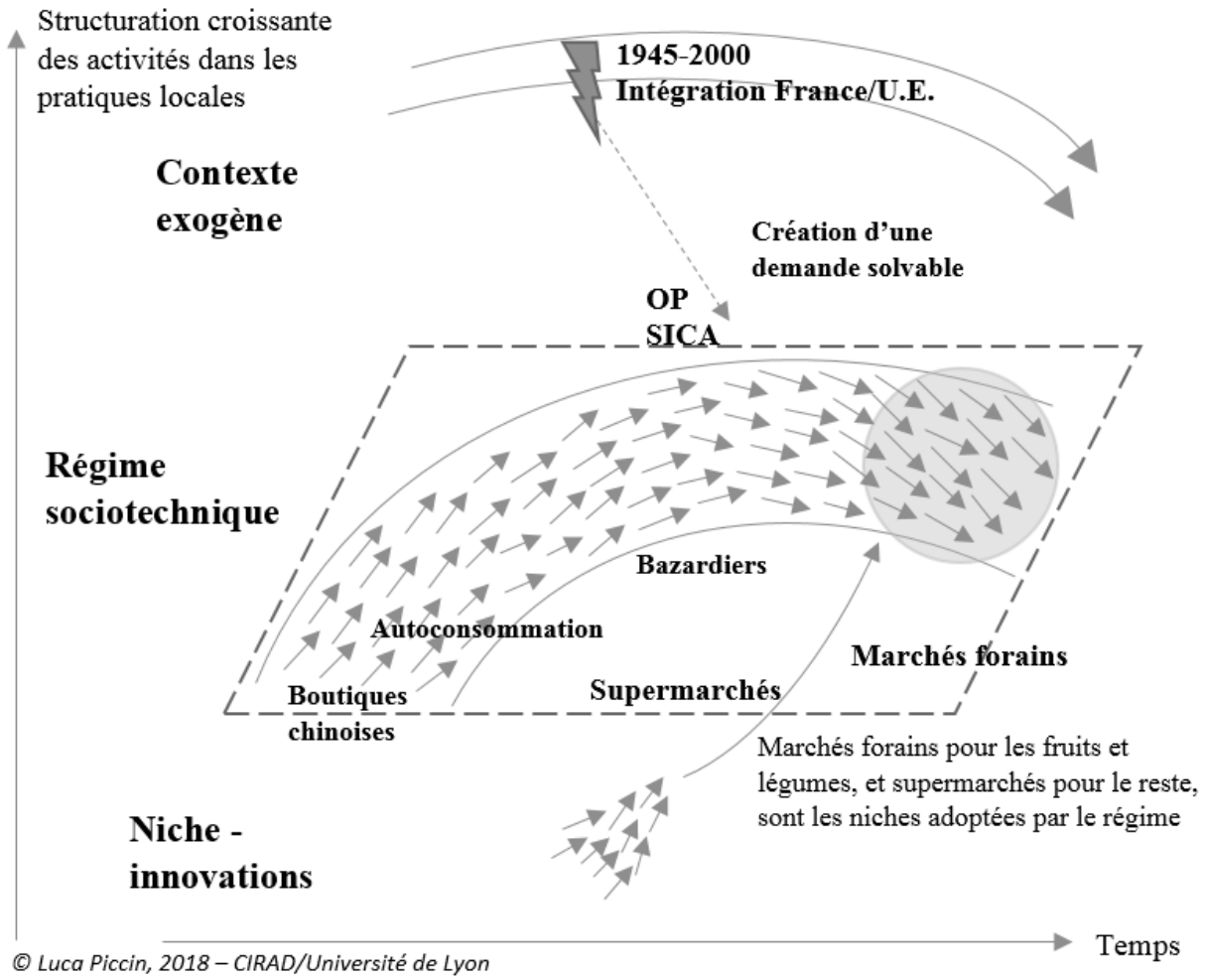

\section{Dynamiques récentes de l'organisation du marché des fruits et légumes}

L'apparition d'une classe moyenne impulsée par la départementalisation a eu pour conséquence la transformation de la boutique en libre-service. Les Chinois profitent de leur présence capillaire et de leur connaissance du secteur du négoce, mais aussi de relais dans des structures institutionnelles comme la Chambre de Commerce, ou en politique via des élus locaux, pour investir ce nouveau mode de commercialisation. Le 2 aout 1961, les Lawson inaugurent le premier Prisunic à Saint-Denis, suivis des TsangKing-Sang qui en 1966 transforment leur libre-service de Saint-Pierre en supermarché sous l'enseigne des Nouvelles Galeries avant de créer un autre magasin toujours à SaintPierre en 1970, puis au Port en 1972. Ils seront rejoints par les grandes familles blanches: les Caillé, commerçants depuis le début du siècle; la famille De Chateauvieux, qui se débarrasse du sucre et crée le groupe Vindémia ; le groupe Hayot, arrivé tardivement et présent aussi aux Antilles (Ho, 2011). Suite à l'introduction du Revenu Minimum d'Insertion en 1989 et son alignement avec la métropole en 1995, qui ont pour effet de stimuler la consommation des ménages plus faibles, ces groupes se livrent à une concurrence féroce qui débouche sur des acquisitions et des changements d'enseignes. Les Chinois savent encore une fois se défendre et parviennent à maintenir des parts de marché malgré la puissance financière des groupes concurrents. L'essor fulgurant des grandes et moyennes surfaces dans les années 1980 et surtout 1990, va de pair avec une augmentation des importations : le taux de couverture annuel en valeur 
chute, passant de $75 \%$ en 1972 à $12 \%$ en 1986, pour se stabiliser autour de $6 \%$ aujourd'hui (Douanes, INSEE).

\title{
Le marché de gros, clé de voute de la commercialisation de fruits et légumes
}

La fin de la SICAMA n'a pas perturbé l'approvisionnement en fruits et légumes, qui se réorganise autour des bazardiers. Cet évènement majeur accélère la création du marché de gros. Créé à Saint Pierre en 1992, il devient société à économie mixte dès 1995 et permet la rencontre d'environ 800 producteurs et 300 bazardiers. Le dispositif va structurer le marché des fruits et légumes :

\begin{abstract}
« Avant, les ventes se faisaient la nuit, « derrière bazar » à Saint-Denis et « derrière bazar » à Saint Pierre ${ }^{17}$. C'était un vrai souk, il y avait de tout, pas que des légumes, des femmes aussi circulaient autour, en plein centre-ville. Avec le marché de gros, tous les bazardiers ont commencé à venir à des heures précises, les producteurs disposent d'un emplacement et les acheteurs aussi. C'est devenu la référence, même la DAF s'appuie sur le marché de gros pour établir ses mercuriales » (Entretien AS, directeur du marché de gros de 1992 à 2017).
\end{abstract}

Figure 8 : Créé le 20 janvier 1992, le marché de gros de Saint Pierre est encore aujourd'hui fréquenté par plus de 800 paysans, en grande majorité originaires du sud de l'lle, berceau des cultures fruitières et maraichères

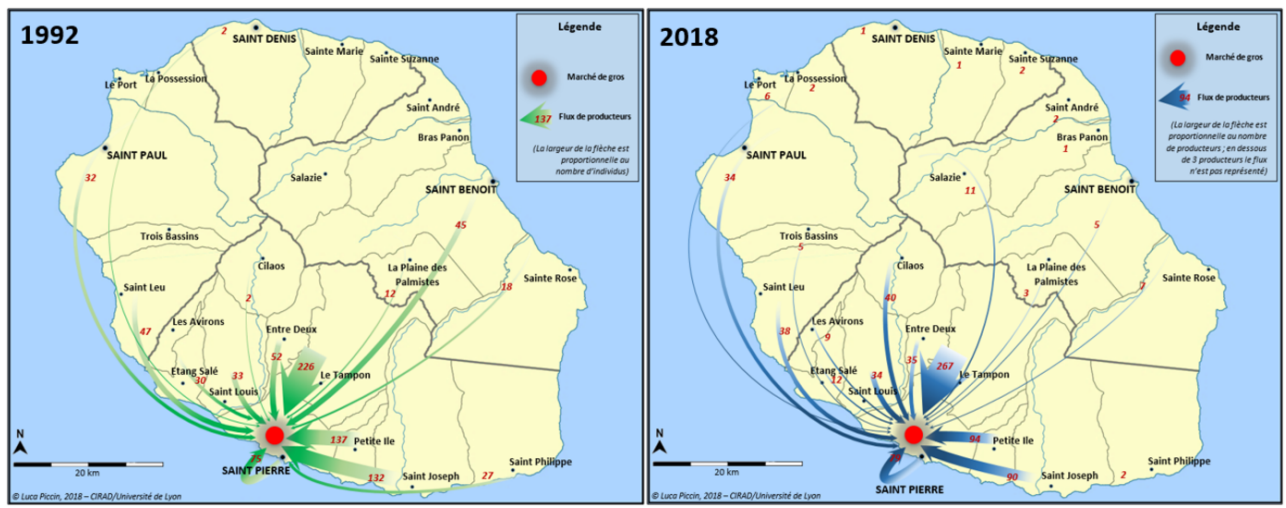

Sources : archives marché de gros

Dans les années 1990, avec la création du marché de gros, la commercialisation s'organise autour de trois pôles: a) collecte par des bazardiers, qui revendent euxmêmes ou cèdent les produits à d'autres détaillants, ou encore font du porte à porte, et qui profitent de la fin de la SICAMA pour s'insérer dans le marché des collectivités ; b) apports par des agriculteurs, par des agriculteurs-bazardiers ou par des bazardiers grossistes sur le marché de gros ; c) vente directe ou via des bazardiers sur les marchés forains et en bord de route. Le marché de gros reste encore aujourd'hui une référence pour toute l'agriculture réunionnaise.

\section{La résistance des bazardiers questionne le soutien public réitéré à l'intégration verticale avec l'aval}

Les années 1990 sont encore plus marqués par les échecs : la SEMEX, société d'économie mixte pour l'exportation est contrainte à fermer en 1995, comme la SICA Victoria qui la 
remplace, la coopérative Tropicafruits créée pour remplacer la SICAMA ferme ses portes en 1997... Le Verger Tropical en 2005. Ces structures collectives n'intéressent qu'une minorité de producteurs : la vente directe est plus rémunératrice, ou alors la vente au bazardier, dont le prix de vente au détail est quasi systématiquement inférieur au prix final du même produit vendu en grande surface. C'est grâce au POSEIDOM et plus particulièrement aux mesures destinées à aider la commercialisation par un soutien direct au prix, que les organisations de producteurs peuvent rester compétitives sur le marché local. Depuis 1995 ces aides du POSEIDOM ont été renforcées et le nombre d'organisations de producteurs est passé de 3 en 2002 à 9 aujourd'hui, tandis que les volumes de fruits et légumes commercialisés par ces structures sont passés de $15 \%$ à $25 \%$ du marché.

Figure 9 : Les circuits de commercialisation actuels

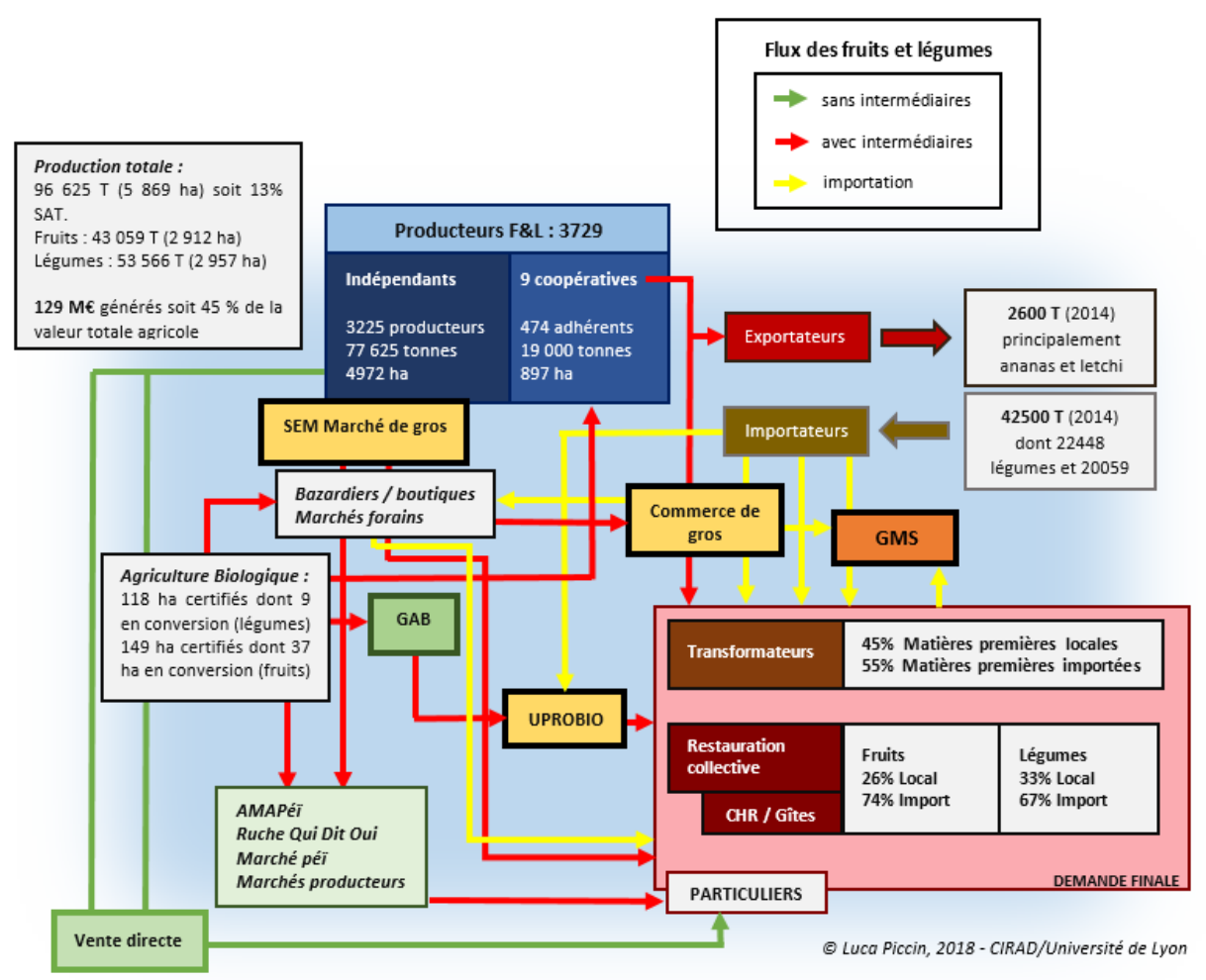

Sources : entretiens, Conseil Départemental

Durant les vingt dernières années l'agriculture réunionnaise s'est alignée sur la tendance européenne à la concentration des structures de production. Les institutions en charge du monde agricole ont encouragé cette voie à travers le POSEIDOM, en privilégiant les aides couplées au volume de production. Ce modèle d'exploitation à temps plein, spécialisée, à forte productivité grâce à la mécanisation, répond à une logique agroindustrielle basée sur la recherche du rendement maximum et de compétitivité du prix afin de répondre aux exigences de la grande distribution et des transformateurs. Ces entreprises en aval dominent l'économie insulaire à travers une «stratégie de prédation » qui les conduit à s'approprier la rente administrative via la consommation finale et à réinvestir en dehors des frontières réunionnaises (Afrique, Maurice, Vietnam) afin de maximiser leurs profits (Dimou, 2004). Les productions locales non périssables tendent alors à être évincées (par exemple: ail, carottes, 
oignons, agrumes) au profit de produits importés. Les productions périssables (maraîchage notamment) sont moins concurrencées par les importations : entre 70 et $80 \%$ des volumes vendus en fruits et légumes ne passent pas par le secteur coopératif adossé à la grande distribution.

La concurrence entre usages multiples de l'espace et l'étroitesse du territoire, dont tous les lieux sont accessibles en moins de deux heures de trajet, favorisent l'écoulement des produits aussi bien dans le commerce de gros que de détail. Cette présence à tous les stades de la mise en marché et la limite floue entre les rôles d'agriculteur et de bazardier sont encore aujourd'hui des caractéristiques centrales de la filière fruits et légumes de La Réunion. Les dispositifs sont donc à la fois hybridés et détournés. Cette coexistence paradoxale entre secteur « informel » et secteur « structuré » est connue et n'est pas une prérogative de La Réunion (Latouche, 1989). De surcroit, elle est bénéfique pour la sécurité alimentaire, puisque agriculteurs et mangeurs ont le choix entre plusieurs circuits d'approvisionnement.

\section{Conclusion}

37 Dans un espace exigu marqué par des inégalités de revenu très fortes, grevé par le poids d'un passé colonial encore vif, le dynamisme dont ont fait et font encore preuve les bazardiers tient à leur appartenance à une société en rupture et marginalisée, que l'assimilation nationale et l'économie de transferts peinent à dissimuler. L'analyse des transitions de l'approvisionnement en fruits et légumes montre que le travail d'intermédiation des bazardiers est fortement imbriqué aux dispositifs institutionnels de développement agricole, avec lesquels ils sont en confrontation permanente. La prégnance contemporaine des bazardiers dans le système alimentaire est donc à chercher dans la marge d'indétermination qui existe depuis longue date entre un ordre de réalité endogène et un ordre de réalité émanant de l'administration. Ce même mécanisme a été relevé par Cheauveau (1985) en Afrique de l'Ouest, où producteurs et distributeurs «informels » détournaient et tiraient profit de projets émanant des structures officielles de l'Etat. Comme dans le cas de La Réunion, ils répondaient aux besoins d'une société civile pourtant soumise à la politique d'une administration nationale qui s'obstinait sans cesse à appliquer des dispositifs de développement localement inadaptés. C'est là, il nous semble, tout l'intérêt d'une approche par les transitions sociotechniques, qui permettent de poser un autre regard sur la modernisation, moins linéaire et plus attentif aux phénomènes de verrouillage et de dépendance du sentier. Restent encore à évaluer les dynamiques contemporaines liées à l'émergence du commerce électronique et de formes marchandes tendant à rapprocher producteurs et mangeurs autour de nouvelles valeurs socio-culturelles et environnementales. 


\section{BIBLIOGRAPHIE}

BENEDICT B., 1958, Cash and Credit in Mauritius, The South African Journal of Economics, 26, 3, p. 213-221.

BENOIST J., 1981, Paysans de La Réunion, Extrait de L'Annuaire des pays de l'Océan Indien, vol. 8.

BERARD L., MARCHENAY Ph., 1994, Ressources des terroirs et diversité bio-culturelle.

Perspectives de recherche, Journal d'agriculture traditionnelle et de botanique appliquée, $36: 2$, p. 87-91.

BERNARD DE RAYMOND A., 2013, Le marché des fruits et légumes en France, Rennes, PUR.

BOUCHET S., 2009, Petite Ile, in GERAUD J.-F., Histoire des communes de la Réunion, vol. 2, La Réunion, Éd. Delphine.

BOSCHMA R., COENEN L., FRENKEN K., TRUFFER B., 2017., Towards a theory of regional diversification: combining insights from Evolutionary Economic Geography and Transition Studies, Regional Studies, 51:1, p. 31-45.

CHALEARD J.-L., MOUSTIER P., LEPLAIDEUR A., 2002, L'approvisionnement vivrier des villes en Guinée : entre fragilité et dynamisme, Autrepart, $3: 23$, p. 5-23.

CHAUVEAU J.-P., 1985, L'avenir d'une illusion. Histoire de la production et des politiques vivrières en Côte-d'Ivoire, Etudes Rurales, 99-100, p. 281-325.

CODER Y., DUPLOUY B., FABRE E., 2016, La départementalisation de La Réunion 1946-2016 : 70 ans de mutations économiques, Insee Analyses, $\mathrm{n}^{\circ} 18$.

DEFOS DU RAU J., 1960, L'île de La Réunion. Etude de Géographie Humaine, Thèse de doctorat, Bordeaux.

DIMOU M., 2004, Economies insulaires et trajectoires de développement. Une comparaison entre Maurice et La Réunion, Région et Développement, 20, p. 215-236.

DUPON J.-F., 1967, Les immigrants indiens de la Réunion. Evolution et assimilation d'une population, Cahiers d'outremer, 77, p. 49-88.

EVE P., 2013, Le garde-manger réunionnais, de son accommodement à sa mise en scène, Revue Historique de l'Océan Indien, 10, p. 44-69.

HANSEN T., COENEN L., 2015, The geography of sustainability transitions: a literature review, Environ. Innov. Soc. Transit. 17, p. 90-107.

HO H. Q., 2004, Histoire économique de l'île de la Réunion (1849-1881), Engagisme, croissance et crise, Paris, L'Harmattan.

HO H. Q., 2008, La Réunion (1882-1960), Histoire Economique, Paris, L'Harmattan.

HO H. Q., 2011, 38 chefs d'entreprises témoignent : Île-de-la-Réunion, 1960-2000 : destins de familles, interculturalité et économie, Saint Denis, Ed. Poisson Rouge.

FITAU A., 1859, La crise alimentaire et l'immigration des travailleurs étrangers à l'île de La Réunion, Questions coloniales, Paris, Typographie Morris et compagnie.

GEELS F.W., SCHOT J.W., 2007, Typology of sociotechnical transition pathways, Research Policy, 36, p. 399-417.

GOUROU P., 1984, Riz et civilisation, Paris, Fayard. 
HUETZ DE LEMPS Ch., 1972. Les Chinois aux Hawaii, Etudes de Géographie Tropicale offertes à Pierre Gourou, Paris, Mouton, p. 183-208.

IRFA, 1979, Recherche agronomique et production fruitière à l'île de La Réunion, Plan directeur de mise en valeur.

JAUZE J.-M., 2000, Villes et patrimoine à La Réunion, L'Harmattan, Université de La Réunion.

LAMINE C., BUI S., OLLIVIER G., 2015, Pour une approche systémique et pragmatique de la transition écologique des systèmes agri-alimentaires, Cahiers de recherche sociologique, 58 , p. 95-118.

LATOUCHE S., 1989, Les paradoxes de la « normalisation » de l'économie informelle, Revue Tiers Monde, XXX, 117, p. 227-233.

LECOMPTE A., 1975, Habitat, dans : Atlas des Départements français d'Outre-Mer. 1. La Réunion, Paris, CNRS - IGN.

LEBLANC F. 2017. Commercer dans la ville, Ethnologie française, 165, (1), 5-10.

LEFEVRE D., 1974, La situation économique de la Réunion au début du VIe Plan, Annales de Géographie, t. 83, 457, p. 319-349.

LUSSAULT M., STOCK M., 2010, Doing with space: towards a pragmatics of space, Social Geography, 5, p. 11-19.

MINTZ S., 1964, Peasant market places and economic development in latin America, The Graduate Center for Latin American Studies, Vanderbilt University, Occasional Paper No. 4.

NICAISE S., 2013, Capab' ensemb'. Une Histoire réunionnaise d'éducation populaire, 1962-2012, Saint André, Océan Ed.

RAMSAMY J.R., 2012, Les travailleurs indiens sous contrat à La Réunion (1848 - 1948) : entre le retour programmé et le début des intégrations, Thèse de Doctorat en Histoire, Université de La Réunion.

WARD B., 1960. Cash or Credit Crops? An Examination of Some Implications of Peasant Commercial Production with Special Reference to the Multiplicity of Traders and Middlemen, Economic Development and Cultural Change, 8:2, p. 148-163.

WONG HEE KAM E., 1996, La diaspora chinoise aux Mascareignes : le cas de La Réunion, L'Harmattan, Université de La Réunion.

\section{NOTES}

1. Programme portant mesures spécifiques dans le domaine de l'agriculture en faveur des régions ultrapériphériques, 2012, Tome 4, Chapitre 5.B. Diversification végétale, pag. 12. Adopté par la Communauté Européenne dès 1989, le Programme d'Options Spécifiques à l'Eloignement et à l'Insularité des DOM (POSEIDOM) comporte des dispositions particulières et des financements en faveur du particularisme des DOM.

2. Terme typique du français de l'océan Indien, qui désigne les légumes dont les feuilles ou les pousses sont mangées cuites.

3. Expression qui qualifie une population issue du peuplement européen, paupérisée mais attachée à une origine blanche, donc libre, qui la distingue de l'affranchi.

4. Le premier bateau à vapeur reliant Saint Paul à Saint Denis a été introduit par la famille Panon-Desbassyns dans les années 1820. Il n'y en avait alors pas plus qu'une dizaine dans toute la 
France métropolitaine. Jusqu'au début du $\mathrm{XX}^{\mathrm{e}}$ siècle le batelage fût un commerce florissant qui permit la réalisation de profits remarquables.

5. Le rôle central des commerçants chinois dans les économies insulaires a aussi été souligné par Ward (1960) à Hong Kong et dans le Sarawak, par Huetz de Lemps à Hawaii (1972) ou par Benedict (1958) à Maurice.

6. Comparable à l'éthique protestante détaillée par Max Weber.

7. Récépissé qui était versé aux planteurs par les sucriers.

8. Dans la région du Tampon, les toponymes des hameaux se basent sur les points kilométriques de la route nationale, ici le $23^{\mathrm{e}} \mathrm{Km}$.

9. En décembre 1971 le salaire minimum est de 22050 francs CFA et le salaire nominal minimal de la fonction publique pour titulaire de 88929 francs CFA. Les traitements des fonctionnaires représentent le quart du PIB (Lefèvre, 1974, p. 324).

10. Au sens de Bérard et Marchenay (1994).

11. Le Quotidien, 5 février 1983.

12. Le Quotidien, 29 janvier 1985.

13. Le Quotidien, 2 juillet 1984. La SICAMA était considérée une "poubelle " en raison de la mauvaise qualité des produits qu'elle commercialisait, au point que nombre d'opérateurs de la restauration scolaire préféraient se fournir auprès de bazardiers grossistes.

14. Le Quotidien, 5 février 1983.

15. Le Quotidien, 23 janvier 1985.

16. Le Quotidien, 10 janvier 1985.

17. «Derrière bazar » signifie à l'arrière du marché couvert, lieu traditionnellement conçu pour la vente des fruits et légumes. Les marchés couverts de Saint Denis, de Saint Pierre et du Port étaient les ancêtres de l'actuel marché de gros.

\section{RÉSUMÉS}

Cet article étudie les processus d'émergence, les évolutions et les adaptations qu'a connus l'approvisionnement alimentaire sur l'île de La Réunion, depuis l'abolition de l'esclavage en 1848, jusqu'à nos jours. Nous identifions deux trajectoires de transition, au sein desquelles coexistent plusieurs modalités d'organisation de l'approvisionnement. L'article explicite comment les transitions se succèdent dans un continuum temporel, malgré des crises majeures qui affectent durablement les stratégies des acteurs en présence. En particulier, nous montrons comment les bazardiers ont été capables de s'adapter à ces transformations et de résister aux tentatives de discipline collective imposées par les autorités, tout en préservant leur emprise dans la mise en marché des fruits et légumes.

This article studies the processes of emergence, evolution and adaptation experienced by the food supply industry of the island of La Reunion between the abolition of slavery in 1848 and today. We identify two main trajectories of transition in which several ways or organising the food supply system co-exist The article explains how transitions succeed one another in a temporal continuum, despite major crises that permanently affect the strategies of the actors involved. In particular, we show how the bazardiers were able to adapt to these transformations and to resist the attempts of collective discipline imposed by the authorities, while preserving their grip on the marketing of fruits and vegetables. 
INDEX

Mots-clés : marchés, géographie des transitions, intermédiaires, fruits et légumes, La Réunion.

Keywords : markets, geography of transitions, intermediaries, fruits and vegetables, La Reunion

\section{AUTEURS}

LUCA PICCIN

Université de Lyon 2, Laboratoire Etudes Rurales et CIRAD, UMR Innovation luca.piccin@cirad.fr

\section{CLAIRE CERDAN}

CIRAD, UMR Innovation claire.cerdan@cirad.fr

\section{CLAIRE DELFOSSE}

Université de Lyon 2, Laboratoire Etudes Rurales claire.delfosse@wanadoo.fr

JEAN PAUL DANFLOUS

CIRAD, UMR Innovation jean-paul.danflous@cirad.fr 